\title{
A Design Method for Complex Fenestration Systems Using Forward Raytracing
}

\author{
Islam Ayman Mashaly ${ }^{1}$, Veronica Garcia-Hansen ${ }^{1}$, Gillian Isoardi ${ }^{1}$, Marianella Chamorro-Koc ${ }^{1}$ \\ ${ }^{1}$ Queensland University of Technology, Brisbane, Australia
}

\begin{abstract}
Complex fenestration systems (CFS) are becoming a part of the building designer's daylight strategies to bring higher quality of daylighting. While numerous tools effectively analyse the behaviour of CFS in the building space, the field lacks a comprehensive CFS design tool that takes the built context and environmental conditions into consideration. The paper proposes a method for designing CFS with their performance optimized considering the building context and environmental conditions. A parametric forward raytracing design tool is presented and the design evaluation criteria for such CFS is developed to assist building practitioners in easily and quickly design CFS. The tool is to reduce the need for learning complicated technologies, outsourcing the design task or breaking a link in the workflow of building designing. A case study is carried out to test the current and potential capabilities of the proposed work-inprogress method. The results show that an optimized CFS design using the forward raytracing tool can be achieved in shorter times with improved performance compare to non-optimized CFS designs.
\end{abstract}

\section{Introduction}

Effective daylighting is one of the valuable strategies that designers aim to achieve when designing buildings. It has a positive physiological effect on occupants occupying the space especially in commercial buildings and office spaces where occupants have to stay indoors for long periods of times (Boyce, Hunter, \& Howlett, 2001; Farley \& Veitch, 2001) (Choi, Beltran, \& Kim, 2012; Joseph, 2006). Moreover, effective daylighting strategies are a useful approach for saving energy in buildings (Gratia \& De Herde, 2003). One way to have adequate daylighting in a space is through the use of complex fenestration systems (Garcia-Hansen, 2006; Isoardi, 2009; Mayhoub, 2014). Complex fenestration systems (CFS) are building assemblies that improve the daylight performance of indoor spaces in building (Jakica, 2017). They are usually characterised to redirect daylight from overexposed space perimeter to deeper areas inside the room, reducing the visual discomfort and increasing the daylight distribution in the space (Kischkoweit-Lopin, 2002). This allows CFS to overtake artificial lighting during day time which increases energy savings. The complex fenestration systems often contain optical elements (such as mirrors, prisms, thin films) that redirect light towards the ceiling and reflect it downwards deeper into the space (Jakica,
2017). Current practices to optimize the daylighting design within the whole building design process are becoming more efficient. Designers tend to use new technologies that implement CAD techniques to easily model, manipulate, and design fenestration systems to optimize the daylight performance within a building space. However, the technology is currently limited when it comes to designing complex fenestration systems (CFS). In order to design a CFS that responds to the building conditions, complicated daylight simulation methods are used (Garcia-hansen, Isoardi, Hirning, \& Bell, 2012; Hirning, Garcia Hansen, \& Bell, 2010)due to the complexity of the simulation techniques needed to simulate the optical behaviour of CFS.

\section{Requirements for Optimizing CFS Design}

In order to design an optimum CFS that enhances the daylight quality within a space a number of important factors are considered. The key factors are the illumination levels, daylight distribution, glare probability, energy consumption levels, and other nontechnical parameters such as cost, maintenance, and availability (Garcia-hansen et al., 2012). While it is improbable to design a fenestration system that recognizes the best of all the factors together, an optimum design that balances between those factors can be reached through an iterative process. When optimizing a CFS, changing certain parameters can drastically affect the outcome of the performance of the CFS. Most of those factors are geometrical parameters that contributes in the redirection and transport of light to certain vectors, while other parameters might include the material surface reflectivity or the material refractive index.

It is also important to note that optimizing the design of a complex fenestration system is different than analysing it, thus the tools and techniques used in analysing CFS can be distinct from the tools required for designing one.

\section{CFS Evaluation and Design}

The performance of a CFS can be measured using a transmission matrix called Bidirectional Scattering Distribution Function (BSDF). A BSDF describes the ray scattering properties of a fenestration system according to the geometry and materials used in it (Ward, Mistrick, Lee, McNeil, \& Jonsson, 2011). The BSDF can be generated by modelling the geometry and assigning the material of the fenestration system and by running it through a raytracing simulation program we can extract the directional properties of the fenestration system. Two 
commonly used raytracing programs that can generate such properties are Radiance's genBSDF command and TracePro.

The CFS bidirectional scattering properties are beneficial for accurate daylighting and thermal simulations, which allow evaluating the CFS annual climatic performance in the building during the design stage within Radiance simulation software, which is a backwards raytracing program (Mcneil, Jonsson, Appelfeld, Ward, \& Lee, 2013). El-Henawy et al., 2014; Hoffmann et al., 2016; Mashaly et al., (2017) were able to simulate CFS on Radiance and evaluate their annual performance on a particular setting. When it comes to tailoring the design of CFS and changing the parameters, it is hard to optimize the design using radiance; since such simulation method can effectively analyse the performance of pre-designed CFS, however, this approach does not allow for optimisation of the CFS tailored to a specific space.

In order to accurately design complex fenestration systems, one should refer back to the fundamentals of light redirection (refraction and reflection). Such technique requires knowledgeable professionals in the fields of optics and have always required familiarity with specialized optical design tools such as TracePro or Zemax to simulate and optimize light redirecting systems (Jakica, 2017). TracePro and Zemax are validated optical design tools that use forward raytracing to simulate the exact optical behaviour of different components such as lenses, prisms and mirrors (Andersen, Rubin, \& Scartezzini, 2003). Mashaly et al., 2017; Mohamed et al., (2014) used the TracePro software in designing, optimizing and measuring the performance of daylight prismatic panels, reflectors and concentrators. (Isoardi, 2009) used Zemax to optimize the design of an optical system created for multistorey buildings in tropical and subtropical environments. However, the drawback to the optical design tools that they are not incorporated with the whole building design process. (Jakica \& Zanelli, 2014) developed a raytracing algorithm specifically for developing complex building integrated photovoltaic (BIPV) systems. The application of the algorithm was mainly on a macro scale.

However, recently a number of researchers started to show interest in finding simpler methods to design CFS responsive to the built environment (McNeil \& Lee, 2012; Tsangrassoulis, Bourdakis, Geros, \& Santamouris, 2006; Tsangrassoulis, Machairas, \& Axarli, 2013; Vera, Uribe, Bustamante, \& Molina, 2017) the advantages and limitations of these methods are discussed in the next section of the paper.

\section{Existing Methods}

In one of the few publications on designing CFS within the building context, Vera, Uribe, Bustamante, \& Molina, (2017) attempted to optimize the shape of a single CFS design. The design process took place with 3 variables in the geometry of the CFS after which they were combined to generate 1,728 BSDF files for all the iterations, and then fed into an optimization engine that uses Radiance that tests the designs the spatial Daylight Autonomy
(sDA) and Annual Sunlight Exposure (ASE), as well as Energy Plus thermal simulation engine that outputs the thermal energy data. The optimum solution for one of the cities they simulated was reached after 35 iterations, which was coordinated through an optimization engine called GenOpt that reads the Radiance and Energy Plus program outputs and using a hybrid Particle Swarm Optimization and Hooke-Jeeves Pattern search (PSO-HJ) (Vera et al., 2017). The average computing time of each simulation was about 3:45 mins that is a total time of 131 minutes, however, it remains unknown how much time was taken to create the 1,728 BSDF files, which can take a much significant amount of time than the main simulation.

The observed limitations of the existing method were:

- Running a number of pre-set iterations of a certain design to measure the performance of a single CFS iteration lacks the flexibility to quickly analyse and change the design according to the building conditions.

- Limited number of solutions.

- Time consuming to simulate the behaviour of a single CFS configuration.

- Design optimization is dependent on holistic factors such as sDA and ASE however it can't include specific factors such as glare; therefore, it is less responsive to the occupant's comfort.

- Knowledge in advanced radiance techniques is needed to simulate and design CFS.

- Unlinked design process from the rest of the building design process.

Another publication by (Tsangrassoulis et al., 2013) presented a simple method to design the profile curve of static specular slat-type systems using GenOpt and a Java Genetic Algorithm Package (JGAP). They developed a $2 \mathrm{D}$ raytracer that simply reflects user-defined equallyweighted parallel rays on the slates curved profile and their weight (ray power) is reduced according to the surface reflectance of the slates. And their ray detection method was done using a semi-circle that calculated the 2D flux transmitted and 2D angular flux distribution exiting the slates system. According to (Tsangrassoulis et al., 2013) only direct solar radiation and specular reflections are considered. Their aim was to reduce summer direct rays while increasing winter rays transmission, increase the view portion of the blinds system and control the redirection of rays by transmitting them upwards.

The observed limitations of the existing method were

- They worked with very simple 2D raytracer that simulates reflection only. Which makes their method only works with simple blind slates.

- Their workflow implies that they designed the slate in their raytracer without accounting for room dimensions or external obstructions.

\section{The proposed method}

The purpose of this research is to propose a new simpler method for designing complex fenestration systems 
(CFS) while considering the building context. This is achieved through reviewing the requirements and the state-of-the-art methods to design CFS and pointing out the gaps and limitations faced. Then a proposed method and tool will be introduced under the name CFStrace, in an attempt to fill the gaps of previous methods along with a parametric forward raytracing tool that supports the method. Afterwards, the method and CFStrace are tested in a case study to comparatively assess its effectiveness and accuracy.

The proposed method is formulated to attempt to address some of the limitations in the existing methods. The proposed method analyses the behaviour of complex fenestrations within the context of a built environment. A forward raytracing technique is used to assess the optical behaviour of complex structures such as prisms, blinds, and louvers. The forward raytracing technique used in CFStrace will only focus on the direct light from the daylight component, since the direct daylight has a major portion of the light power needed to design accurate and representative CFS. And the performance criteria are set around the direct sun rays' power and points of incidence. That being said, the complex fenestrations' form is changed through parametric modelling of the geometry's 3-dimensional form.

In order to achieve that, Grasshopper for Rhino is used, which is a parametric modelling visual scripting tool that allows parametric geometry modification using simple sliders and visual programming. Grasshopper has proven to be a reliable tool in parametric design and optimization of daylight problems (Eltaweel \& SU, 2017; Wagdy \& Fathy, 2015). The geometry was modelled using parametric scripting on Grasshopper. The geometry's represented as Breps that is linked to a raytracing tool developed using python programming language (Jakica \& Zanelli, 2014). The raytracing technique used is a forward raytracing technique that uses a deterministic model where the rays hitting a surface split and follows specular paths (Jakica \& Zanelli, 2014). The raytracing is done using optics physics laws: Snell's law for determining the direction of the ray's output and Fresnel law for defining the intensity of transmitted rays as well as reflected rays. Afterwards the raytraced light enters inside the room and bounces of the surfaces of the walls and ceiling to be collected at the working plane for daylight and performance analysis.

The raytracing process is as follows (Figure 2):

1. Sunlight rays are rendered incident on the plane of the fenestration system (vector direction depending on the position of the sun).

2. Sunlight rays passing through the obstacles outside the room will be omitted from the calculation.

3. The optical properties (such as the refractive index or the reflectivity of materials used) of the CFS will determine the trajectory of the rays while entering the space.
4. The power of reflected rays will be determined by the reflective properties of the materials used in the room's inner walls.

5. The number and power of incident rays per area in the room will be calculated on a specified working plane.

Advantages of proposed method:

1. Less steps to reach an optimum solution.

2. Less scientific knowledge is needed to design CFS, architects and building engineers can use the method and tools to design new CFS.

3. Time saving.

4. New computer-generated (generative) design options which makes flexible designs custommade for different locations.

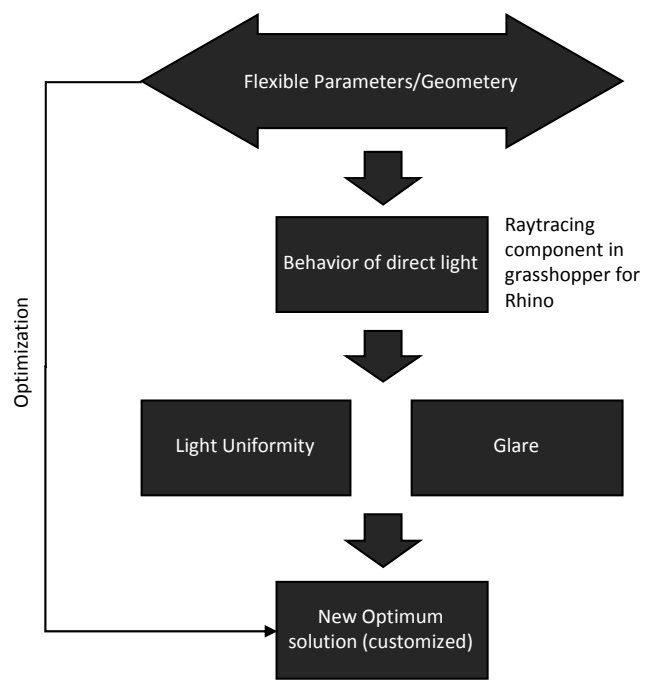

Figure 1: The proposed CFS design workflow.

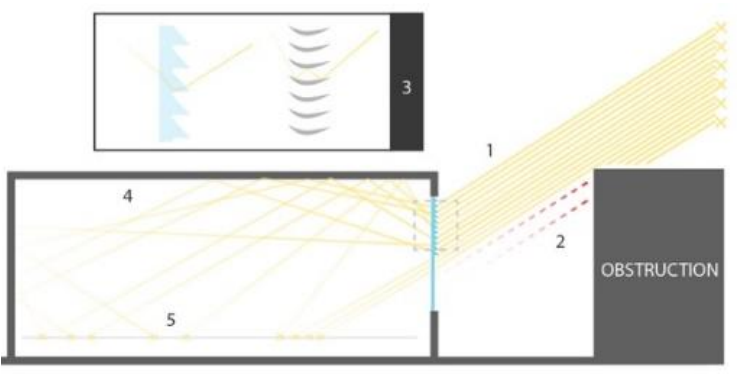

Figure 2: Conceptual steps of the raytracing technique with an obstruction.

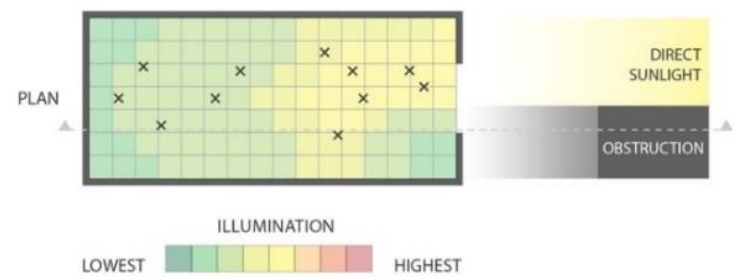

Figure 3: Conceptual plan view of the raytracing technique shown above in Figure 2.

\section{Validation of the raytracing tool}

The raytracing tool, CFStrace, was validated against TracePro, one of the familiar and validated forward 
raytracing software. Mashaly, Nassar, \& El-Haggar (2018) used TracePro to validate their mathematical model for light redirecting panels design in order to ensure the output ray angles are accurate. TracePro uses the Monte Carlo method to simulate light diffraction and light scattering, where random directions are chosen for the rays through either scattering or diffraction processes.

To ensure accuracy in the comparison between TracePro and CFStrace, a model with a sine wave light redirecting panel was selected and created on grasshopper and imported to TracePro to test the validity of the raytracing algorithm. Eight different solar altitudes were tested for both models $\left(10^{\circ}\right.$ to $\left.80^{\circ}\right)$ with 130 rays of equal power directed towards the window area and 45 rays within the total number of rays are directed towards the panel. Around $88-90 \%$ of the rays in both models were directed in a similar direction the other $10-12 \%$ of the rays were directed at different angles due to some randomness in ray splitting since TracePro uses the Monte-Carlo method and CFStrace's raytracing component uses a deterministic method in raytracing. Figure 4 shows the comparison between the TracePro and CFStrace.

Furthermore, tables 1 and 2 shows the results of the number of rays incident and the total power incident respectively on the floor and ceiling resulting from the panel redirection. The root mean square errors (RMSE) are calculated and shown in the tables.

\section{Consideration of the external context (environment)}

The bounds of the dynamic ray trace simulation depend on the sun path diagram of the simulated space location and external obstructions (such as other buildings and the own building's structure that might cause self-shading in some areas).

To ensure the annual sun's positions are being covered, rays are emitted towards the fenestration system every $10^{\circ}$ degrees. The angles created will be according to the boundaries of the sun path diagram of the selected location. And depending on the required optimization criteria, certain angles will be included in the simulation process.

Table 1: Comparison of the number of rays incident on the surfaces and the RMSE between TracePro and CFStrace.

\begin{tabular}{|c|c|c|c|c|c|c|c|c|c|c|}
\hline & & \multicolumn{8}{|c|}{ Number of rays incident } & \multirow[b]{2}{*}{ RMSE } \\
\hline & & $10 \mathrm{deg}$ & $20 \mathrm{deg}$ & $30 \mathrm{deg}$ & $40 \mathrm{deg}$ & $50 \mathrm{deg}$ & $60 \mathrm{deg}$ & $70 \mathrm{deg}$ & $80 \mathrm{deg}$ & \\
\hline \multirow{2}{*}{ Floor } & TracePro & 34 & 40 & 46 & 38 & 10 & 10 & 3 & 13 & \multirow{3}{*}{3.5} \\
\hline & CFStrace & 39 & 45 & 48 & 36 & 9 & 5 & 5 & 10 & \\
\hline \multirow{3}{*}{ Ceiling } & difference & 5 & 5 & 2 & -2 & -1 & -5 & 2 & -3 & \\
\hline & TracePro & 11 & 6 & 2 & 1 & 9 & 19 & 27 & 37 & \multirow{3}{*}{2.3} \\
\hline & CFStrace & 10 & 4 & 3 & 1 & 9 & 21 & 32 & 40 & \\
\hline & difference & -1 & -2 & 1 & 0 & 0 & 2 & 5 & 3 & \\
\hline
\end{tabular}

Table 2: Comparison of the total power incident on the room surfaces and the RMSE between TracePro and CFStrace.

\begin{tabular}{|c|c|c|c|c|c|c|c|c|c|c|}
\hline & & \multicolumn{8}{|c|}{ Power } & \multirow[b]{2}{*}{ RMSE } \\
\hline & & $10 \mathrm{deg}$ & $20 \mathrm{deg}$ & $30 \mathrm{deg}$ & $40 \mathrm{deg}$ & $50 \mathrm{deg}$ & $60 \mathrm{deg}$ & $70 \mathrm{deg}$ & $80 \mathrm{deg}$ & \\
\hline \multirow{2}{*}{ Floor } & TracePro & $63 \%$ & $74 \%$ & $82 \%$ & $46 \%$ & $16 \%$ & $6 \%$ & $4 \%$ & $7 \%$ & \multirow{3}{*}{$3.2 \%$} \\
\hline & CFStrace & $66 \%$ & $77 \%$ & $83 \%$ & $44 \%$ & $10 \%$ & $5 \%$ & $4 \%$ & $2 \%$ & \\
\hline & difference & $2 \%$ & $3 \%$ & $2 \%$ & $-2 \%$ & $-6 \%$ & $-1 \%$ & $0 \%$ & $-5 \%$ & \\
\hline \multirow{2}{*}{ Ceiling } & TracePro & $16 \%$ & $4 \%$ & $4 \%$ & $2 \%$ & $15 \%$ & $33 \%$ & $62 \%$ & $67 \%$ & \multirow{3}{*}{$2.1 \%$} \\
\hline & CFStrace & $13 \%$ & $5 \%$ & $2 \%$ & $1 \%$ & $15 \%$ & $37 \%$ & $62 \%$ & $69 \%$ & \\
\hline & difference & $-3 \%$ & $1 \%$ & $-2 \%$ & $-2 \%$ & $0 \%$ & $4 \%$ & $0 \%$ & $2 \%$ & \\
\hline \multirow{2}{*}{ Total } & TracePro & $80 \%$ & $78 \%$ & $85 \%$ & $48 \%$ & $31 \%$ & $40 \%$ & $67 \%$ & $73 \%$ & \multirow{3}{*}{$2.8 \%$} \\
\hline & CFStrace & $79 \%$ & $82 \%$ & $85 \%$ & $48 \%$ & $25 \%$ & $42 \%$ & $66 \%$ & $71 \%$ & \\
\hline & difference & $-1 \%$ & $4 \%$ & $0 \%$ & $0 \%$ & $-5 \%$ & $2 \%$ & $0 \%$ & $-3 \%$ & \\
\hline
\end{tabular}

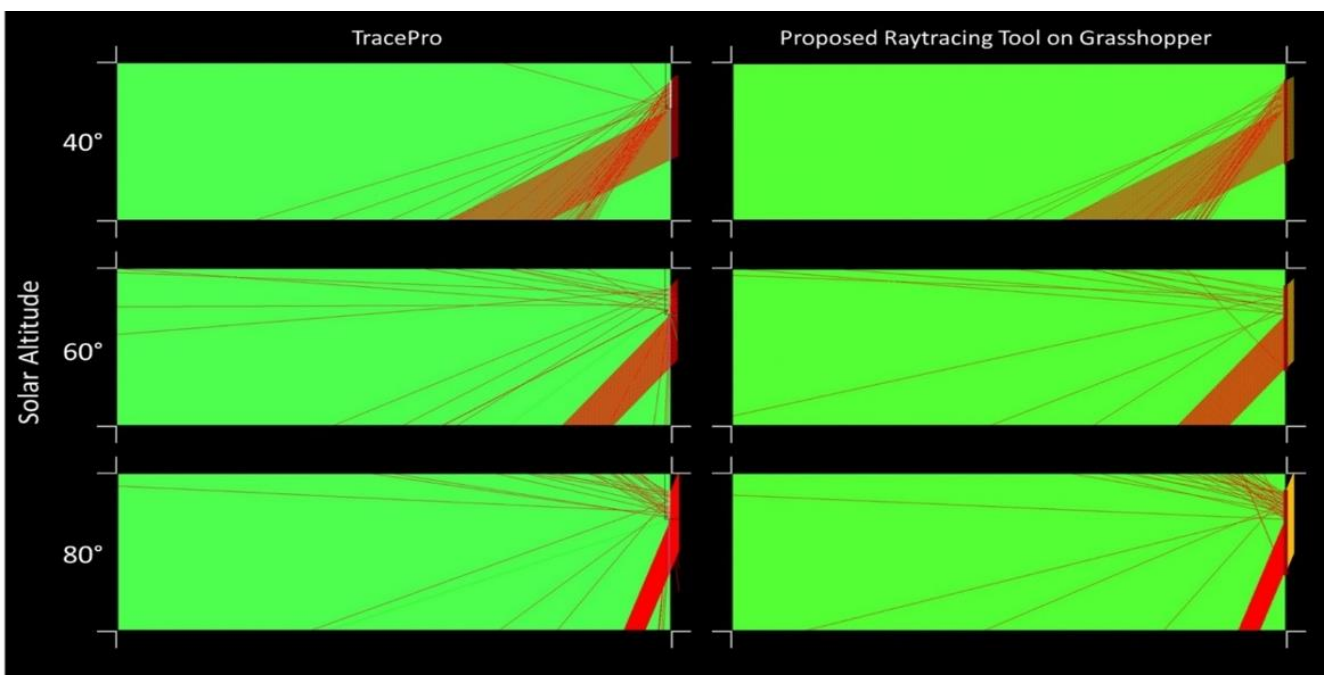

Figure 4: Sample of the TracePro Results vs. the grasshopper raytracing script for the same. 


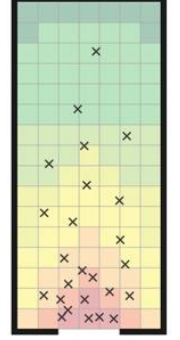

(A)
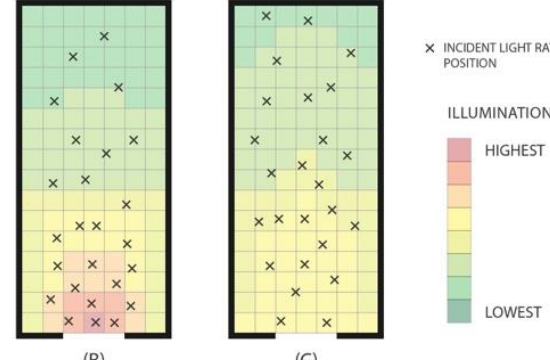

Figure 5: Illustrated example of how the target of uniformity is met at a single point in time.

\section{Optimization}

An objective function of achieving uniform daylighting throughout the depth of the space as well as eliminating glare at the occupant's workspace is being met through optimizing the complex fenestration's geometry.

To achieve uniform lighting the number of rays per square area is automatically counted and matched against the counted rays in different areas on the room's working plane.

\section{Case study \\ Setting}

A case study has been carried out to check the integrity of the proposed workflow and test the created tool's progress. The case study is done on a virtual mock up room (called the reference office) that is used in daylight simulation and comparative analyses of different façade technologies (Reinhart, Jakubiec, \& Ibarra, 2013). The room illustrates the perfect example of an under lit room at the depth and well daylight at the window perimeter creating a "cave effect" as shown in Figure 6.

A performance criteria have been set to optimize the design of a CFS at multiple key dates to cover the sun positions at different times of the year for Brisbane, Qld. Key dates such as the summer and winter solstice and spring or autumn equinoxes are considered in the optimization process to cover the high, mid and low sun angles. Light redirecting panels (LRP) were selected to replace the clearstory panels of the fenestration system, see Figure 8.

\section{Optimization criteria}

An evolutionary solver (Galapagos for Grasshopper) is used to find the optimized solution. Galapagos works on maximizing the value of the objective function (a.k.a. fitness function) given by changing the parameters of the redirecting panel geometry. The geometry of the redirecting panels is the key parameter to achieve the maximization in the fitness function. The parameters are defined as the Genomes and in this case study they were limited to the number of waves (or the distance of on full cycle or period), justification of the maximum amplitude point, and the amplitude of the waves, figure 10 illustrates the $i$. And the objective function is the number of rays incident on the working plane of the room and their position from the window.

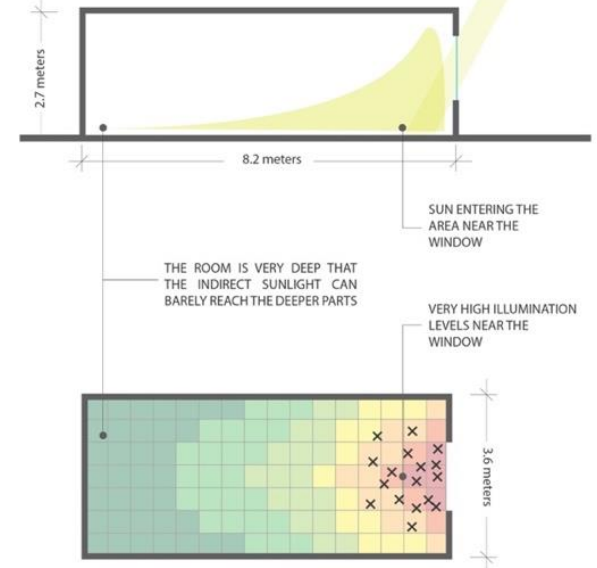

Figure 6: The "Cave Effect" phenomena in the depth of the reference office room.

The equation below summarizes the equation used as the fitness function.

$$
f(x)=N_{\text {rays }}+\sum P_{\text {ray }} \times d_{\text {win }}
$$

$\mathrm{N}_{\text {rays }}=$ number of rays incident on the working plane,

$\mathrm{P}_{\text {ray }}=$ Power of incident ray,

$\mathrm{d}_{\mathrm{win}}=$ distance from window factor.

Figure 7 illustrates the 3 genomes used in the optimization process and Figure 12 illustrates the objective function used as the fitness function in the optimization process.

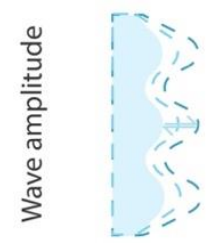

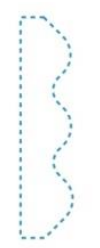

$0 \mathrm{~mm}$
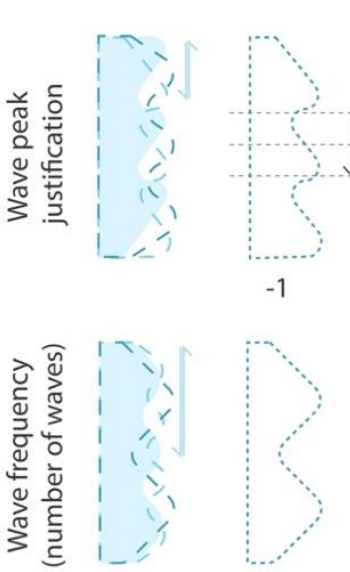

$130 \mathrm{~mm}$

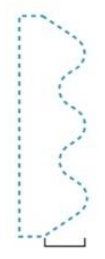

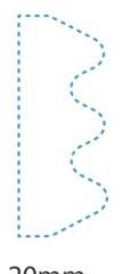

$20 \mathrm{~mm}$
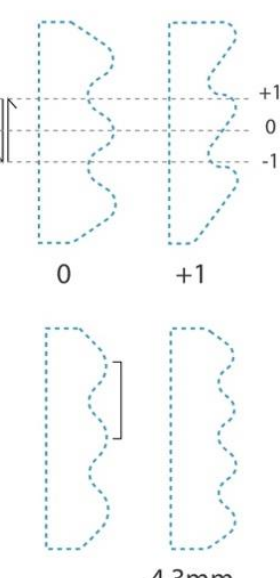

$4.3 \mathrm{~mm}$
Figure 7: Geometry manipulation variables and their constrains.

\section{Comparative analysis of the designed CFS using Radiance's 3 phase method}

After the optimum design was selected, further analysis took place on Radiance to validate the optimum CFS 
performance. Annual illuminance measures were taken for 2 cases:

- Case 1 with an initial CFS design that is not optimized. The non-optimized CFS was selected from previous literature by (El-Henawy et al., 2014)

- Case 2 with the optimized CFS according to the conditions set.

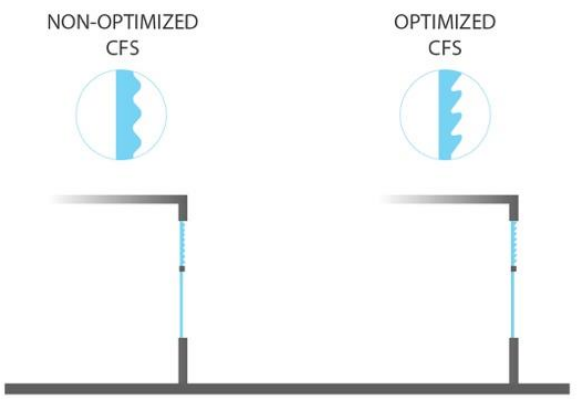

Figure 8: The 2 cases that are compared to each other using the 3-phase method.

The measurements were taken at sensor points set up all around the room's working plane at a $72 \times 74 \mathrm{~cm}$ grid.

\section{Results \& Discussion}

In the proposed raytracing tool, the optimum CFS for the specific settings for Brisbane and room orientation is reached after 80 simulations. While each simulation took around 9 seconds, meaning that the optimum solution was obtained after 12 minutes. The simulations were performed on a desktop computer with an 8 -core Intel® Core $^{\mathrm{TM}}$ i7-6700 processor clocked at a maximum of 3.4 $\mathrm{GHz}, 16 \mathrm{~GB}$ RAM, 1 TB hard drive, an Intel ${ }^{\circledR} \mathrm{HD}$ Graphics 530 Card and a Windows@ 7 Enterprise 64-bit operating system. The most significant illuminance measure comparison was the annual illuminance at the sensor point closest to the window and the sensor point further from the window. As shown in Figure 9, at the sensor near the window the optimized CFS showed decrease in the illumination levels throughout most of the year at $12 \mathrm{PM}$ noon, with the values decreasing the most at autumn and spring seasons. While at summer the effect of using an optimized CFS is not significant and in some rare occasions the optimized CFS would have higher illumination values, however only up to $2 \%$ increase. Whereas at the sensor point deep inside the room, as illustrated in Figure 10, the optimized CFS seems to have up to $60 \%$ increase in illumination levels at autumn and spring periods, while it declines to values slightly above $10 \%$ in summer and around $20 \%$ in winter periods.

This means that the optimized CFS managed to increase illumination levels deep inside the room, while decreasing the lighting near the window at most of the time of the year. This result means the optimized CFS can achieve more daylight uniformity, especially at spring and autumn periods. Around $50 \%$ of the time the lighting has improved by $30 \%$ or more at the node deep inside the room, while light levels decreased near the window by $10 \%$ in $60 \%$ of the time.
It is important to notice that the area just under the window will always have high illumination levels since direct sunlight enters through the clear glass window pane.

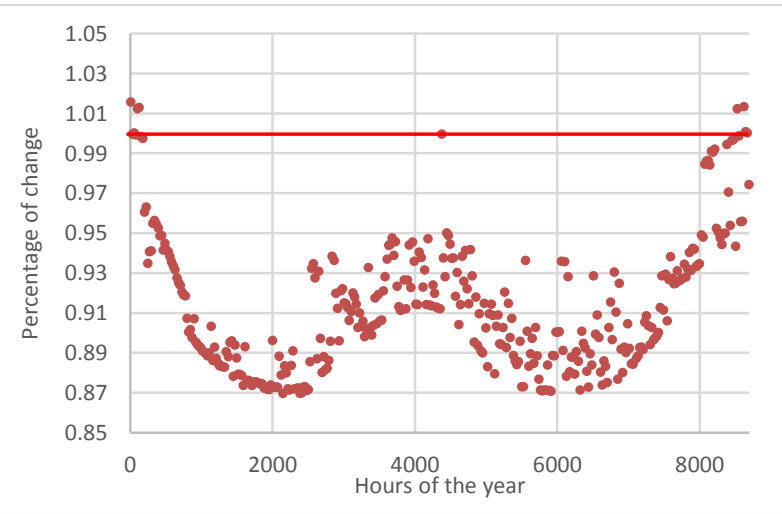

Figure 9: Change done by using an Optimized CFS rather than a non-optimized CFS (Near the window).

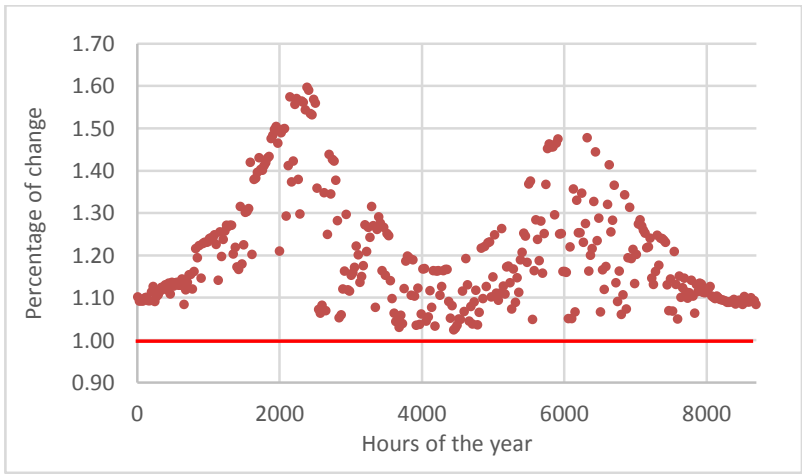

Figure 10: Change done by using an Optimized CFS rather than non-optimized CFS (Deep inside the room).

As shown in Figure 11 \& Figure 13, the overall quality of daylight improved using the optimized design throughout the area of the room. The under lit area (illuminance readings below 300 lux) crawled back deeper inside the room by 1.5 meters as it experienced around $30 \%$ to $60 \%$ increase in daylight. Moreover, the area near the window exhibited around $13 \%$ decrease in illumination.
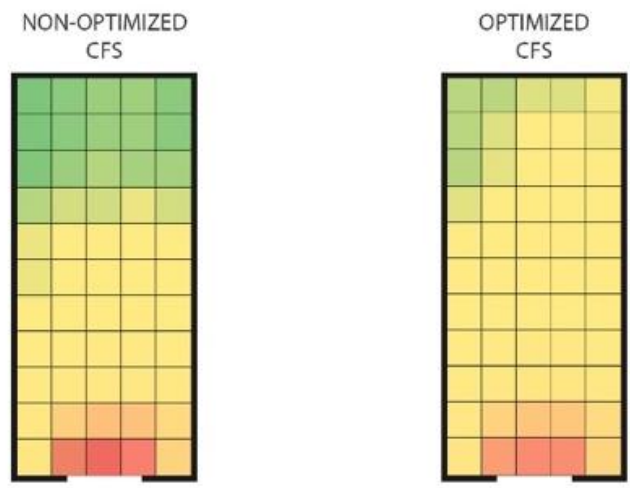

Figure 11: Illumination readings at the $21^{\text {st }}$ of September at 12 PM noon. 


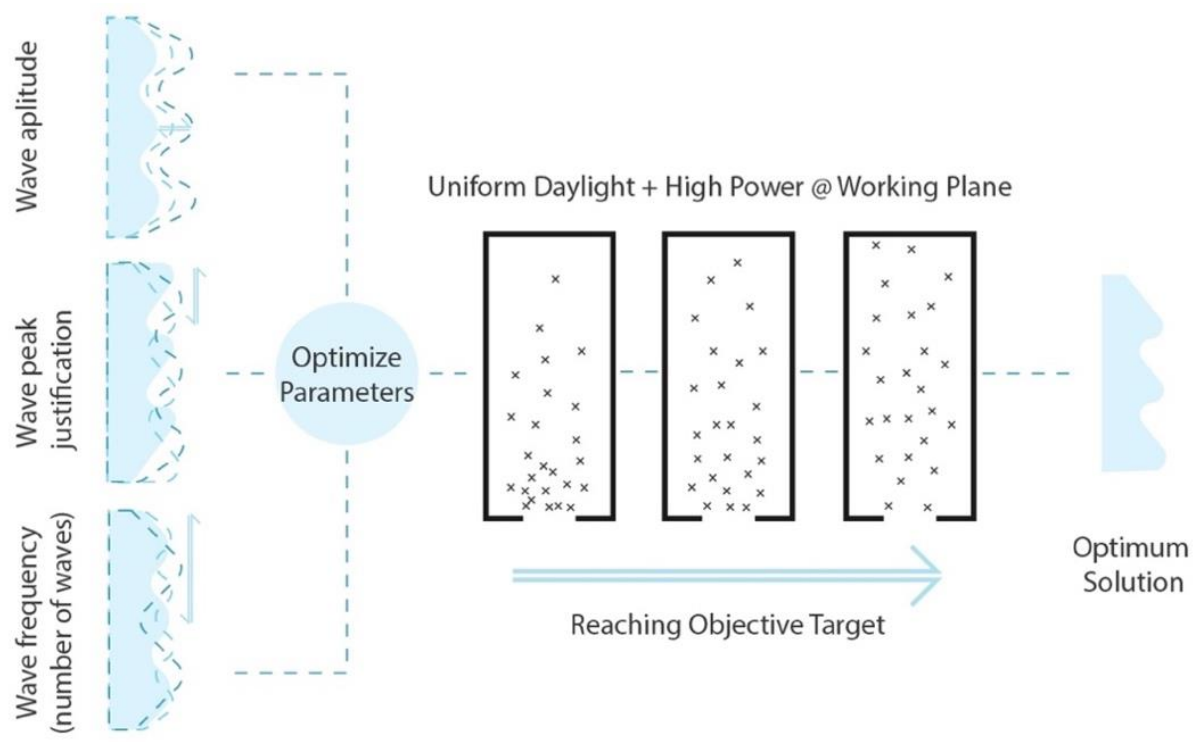

Figure 12: The optimization Diagram.

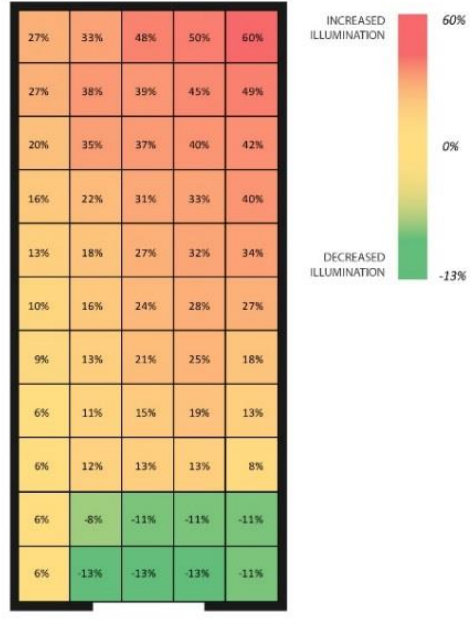

Figure 13: Improvement percentage when using the optimized CFS at the 21 st of September at 12 PM noon.

\section{Limitations to the case study}

Although the performance criteria positively affected the daylight performance for the specified date. Further annual analysis should be carried out after the scripting for taking annual performance into consideration is done. An algorithm will be developed to intake the weather data of the targeted location and extract the necessary information to map the required angle of the sun, its corresponding solar power and cloud coverage which will give a weight to each sun position. Since simulating the 8760 hours per year will seem to be a heavy computational task to carry on, a compromise has to be made and an average value of each major sun angle in increments of 5 or 10 degree will be calculated. This will give a limited number of sun angles, depending on the sun path diagram, that can be included into the optimization simulation process. Other performance criteria will be developed and considered whilst developing the workflow and tools to optimize CFS and other parameters will be tested to result in better performances for the CFS. Only LRPs with certain constrains have been used in the case study as a proof of concept of the workability of the proposed method and tool. Further types of CFS will be tested and more comprehensive analysis will be done to compare and test different strategies and CFS. The inner room boundaries reflection components will be developed, although the reflectivity of surfaces is already considered, for further development the scattering of specular rays will be taken into consideration.

\section{Conclusion}

A new proposed method for designing CFS is presented along with a parametric raytracing tool. The case study implementing the method indicated significant improvement in the time needed to optimize the parameters of the light redirecting panel to redirect light inside the reference office room at a specific setting (time, location, and orientation). Moreover, the CFS optimized had better daylight results than a non-optimized CFS in terms of illumination levels and daylight uniformity. Ultimately, the proposed work-in-progress method offers a solution to improve the daylighting inside buildings by offering optimized CFS design options to increase illumination, have uniform daylighting inside the space, and reduce the glare to the occupants. The outcome of the case study points out that using CFS improves the daylighting. Additionally, the method for designing and analysing such systems would differ than the approach taken to design and optimizing simple daylight fenestration systems.

\section{References}

Andersen, M., Rubin, M., \& Scartezzini, J. L. (2003). Comparison between ray-tracing simulations and bidirectional transmission measurements on prismatic glazing. Solar Energy, 74(2), 157-173. 
Boyce, P., Hunter, C., \& Howlett, O. (2001). The benefits of daylight through windows. In Light. Res. Cent. Retrieved from http://thedaylightsite.com/ wpcontent/uploads/papers/DaylightBenefits.pdf

Choi, J.-H., Beltran, L. O., \& Kim, H.-S. (2012). Impacts of indoor daylight environments on patient average length of stay (ALOS) in a healthcare facility. Building and Environment, 50, 65-75.

El-Henawy, S. I., Mohamed, M. W. N., Mashaly, I. A., Mohamed, O. N., Galal, O., Taha, I., ... Safwat, A. M. E. (2014). Illumination of dense urban areas by light redirecting panels. Optics Express, 22(S3), A895.

Eltaweel, A., \& SU, Y. (2017). Parametric design and daylighting: A literature review. Renewable and Sustainable Energy Reviews, 73, 1086-1103.

Farley, M. J., \& Veitch, J. A. (2001). A Room with a View: A Review of the Effects of Windows on Work and WellBeing.

Garcia-Hansen, V. (2006). Innovative daylighting systems for deep-plan commercial buildings. Faculty of Built Environment \& Engineering, QUT.

Garcia-hansen, V., Isoardi, G., Hirning, M., \& Bell, J. (2012). An Assessment Tool for Selection of Appropriate Daylighting Solutions for Buildings in Tropical and Subtropical Regions : Validation Using Radiance Simulation. Wref, 1-8.

Gratia, E., \& De Herde, A. (2003). Design of low energy office buildings. Energy and Buildings, 35, 473-491.

Hirning, M., Garcia Hansen, V., \& Bell, J. (2010). Theoretical comparison of innovative window daylighting devices for a sub-tropical climate using Radiance. In Proceedings of Improving Energy Efficiency in Commercial Buildings (IEECB) (p. 10).

Hoffmann, S., Lee, E. S., Mcneil, A., Fernandes, L., Vidanovic, D., \& Thanachareonkit, A. (2016). Balancing daylight, glare, and energy-efficiency goals: An evaluation of exterior coplanar shading systems using complex fenestration modeling tools. Energy and Buildings, 112, 279-298.

Isoardi, G. (2009). The Design and Testing of a Daylighting Device: Optimizing the energy and optical performance of Australian commercial buildings. Queensland University of Technology.

Jakica, N. (2017). State-of-the-art review of solar design tools and methods for assessing daylighting and solar potential for building integrated photovoltaics. Renewable and Sustainable Energy Reviews, (July).

Jakica, N., \& Zanelli, A. (2014). Dynamic Visualization of Optical and Energy Yield Co-Simulation of New Generation BIPV Envelope in Early Design Phase Using Custom Ray Tracing Algorithm in Python. 9th Energy Forum, (October).

Joseph, A. (2006). The Impact of Light on Outcomes in Healthcare Settings. Retrieved from www.rwjf.org.
Kischkoweit-Lopin, M. (2002). An overview of daylighting systems. Solar Energy, 73(2), 77-82.

Mashaly, I. A. I. A., Nassar, K., \& El-Haggar, S. (2018). Mathematical model for designing a light redirecting prismatic panel. Solar Energy, 159, 638-649.

Mashaly, I. A., Nassar, K., El-Henawy, S. I., Mohamed, M. W. N., Galal, O., Darwish, A., ... Safwat, A. M. E. (2017). A prismatic daylight redirecting fenestration system for southern skies. Renewable Energy, 109, 202-212.

Mayhoub, M. S. (2014). Innovative daylighting systems' challenges: A critical study. Energy and Buildings, 80, 394-405.

Mcneil, A., Jonsson, C. J., Appelfeld, D., Ward, G., \& Lee, E. S. (2013). A validation of a ray-tracing tool used to generate bi-directional scattering distribution functions for complex fenestration systems. Solar Energy, 98, 404-414.

McNeil, A., \& Lee, E. S. (2012). Using Radiance and GenOpt to Design Static Daylight Redirection Systems. Berkeley, CA.

Mohamed, M. W. N., Mashaly, I. A., Mohamed, O. N., El-Henawy, S. I., Galal, O., Taha, I., ... Safwat, A. M. E. (2014). Light redirecting system using sine-wave based panels for dense urban areas. In Proceedings of SPIE - The International Society for Optical Engineering (Vol. 9191, pp. 1-8).

Reinhart, C. F., Jakubiec, J. A., \& Ibarra, D. (2013). Definition Of A Reference Office For Standardized Evaluations Of Dynamic Façade And Lighting Technologies. 13th Conference of IBPSA, 3645-52.

Tsangrassoulis, A., Bourdakis, V., Geros, V., \& Santamouris, M. (2006). A genetic algorithm solution to the design of slat-type shading system. Renewable Energy, 31, 2321-2328.

Tsangrassoulis, A., Machairas, V., \& Axarli, C. (2013). Simplified Design of a Specular Slat Profile Curve Using 2 Ray Tracing and Genetic Algorithms. In Proceedings of Building Simulation BS2013. Chambery, France: International Building Performance Simulation Association (IBPSA).

Vera, S., Uribe, D., Bustamante, W., \& Molina, G. (2017). Optimization of a fixed exterior complex fenestration system considering visual comfort and energy performance criteria. Building and Environment, 113, 163-174.

Wagdy, A., \& Fathy, F. (2015). A parametric approach for achieving optimum daylighting performance through solar screens in desert climates.

Ward, G., Mistrick, R., Lee, E. S., McNeil, A., \& Jonsson, J. (2011). Simulating the daylight performance of complex fenestration systems using bidirectional scattering distribution functions within radiance. LEUKOS - Journal of Illuminating Engineering Society of North America, 7(4), 241-261. 